\title{
Caracterización morfológica y Evaluación clínica de sustitutos óseos de origen porcino de la casa 3Biomat para su aplicación en lesiones óseas bimaxilares
}

\author{
Morphological characterization and clinical evaluation of porcine bone \\ substitutes of the 3Biomat house for its application in bimaxillary bone \\ lesions
}

Julian Gallón Nausa' ${ }^{\text {, Diego Ernesto Castro Haiek }}{ }^{2}$

\section{Resumen}

Objetivo. Realizar la caracterización morfológica y la evaluación clínica de sustitutos óseos de origen porcino, como material para la regeneración y reconstrucción de tejido óseo afectado en lesiones óseas bimaxilares. Método. Se analizaron características morfológicas de los materiales para regeneración (dispositivos médicos implantables) Biomec Cxy Biomod, el primero es una membrana acelular de submucosa intestinal y el segundo es sustituto óseo particulado y desmineralizado, ambos de origen porcino, por medio de técnicas de coloración y microscopía, con el objetivo de ver celularidad, organización de las fibras colágenas y tamaños de las partículas. Adicionalmente, se realizó un estudio de tipo comparativo prospectivo, con una muestra intencional de 31 pacientes, en los que se hicieron un total de 83 implantes. Los procedimientos quirúrgicos efectuados fueron: implante pos exodoncia más injerto óseo, implante dental e injerto óseo en el mismo tiempo quirúrgico e injerto óseo con un tiempo de 4 meses de integración para la colocación de implantes dentales. Para la evaluación del efecto regenerativo en los diferentes procedimientos quirúrgicos se interpretaron controles clínicos y radiográficos en los que se miró el comportamiento de la cresta ósea marginal. También se realizaron análisis histológicos de biopsias de tejido óseo regenerado. Resultados. En la caracterización morfológica se encontró que los materiales para regeneración son acelulares. La matriz formada por las fibras de colágeno del sustituto Biomec CX presenta una organización y espacios intersticiales que permiten una buena migración celular. El producto Biomod mostró una densidad y tamaño de partícula adecuada para el proceso regenerativo. En la evaluación clínica, todas las diferencias (mesial y distal) de los meses evaluados en relación con la ganancia de hueso, son estadísticamente significativas; prueba t pareada, $\mathrm{p}<0,01$ y ANOVA. El aumento promedio en 4 meses fue $0,235 \mathrm{~mm}$ por mesial $(57,3 \%)$ y $0,237 \mathrm{~mm}$ por distal $(56,7 \%)$. Conclusiones. A partir de los resultados obtenidos tanto en las características morfológicas como en la evaluación clínica de los sustitutos Biomec CX y Biomod, se comprobó que son materiales aptos para su uso en procesos regenerativos en los que se presenten lesiones óseas bimaxilares.

Palabras claves: Regeneración ósea, Sustituto óseo, Osteoconductivo, Osteoinductivo, Lesión ósea.

1. Especialista en Cirugía Oral. Magister Ciencias Biológicas Pontificia Universidad Javeriana, Docente Hospital San Blas servicio Maxilo Facial Correspondencia: j.gallon@javeriana.edu.co

2. Especialista en Patología y Cirugía Bucal. Coordinador Académico Especialización Patología y Cirugía Bucal Pontificia Universidad Javeriana. Correspondencia: castro.d@javeriana.edu.co 


\begin{abstract}
Objective. To perform the morphological characterization and clinical evaluation of porcine bone substitutes as a material for the regeneration and reconstruction of affected bone tissue in bimaxillary bone lesions. Method. Morphological characteristics of Biomec Cx and Biomod (implantable medical devices) regeneration materials were analyzed, the first is an acellular membrane of the intestinal submucosa and the second is a particle and demineralized bone substitute, both of porcine origin, by techniques of coloration and microscope with the objective of search the cellularity, organization of collagen fibers and particle sizes. Then a prospective comparative study was conducted with an intentional sample of 31 patients in which a total of 83 implants were made. The surgical procedures were: Post-extraction implant plus bone graft, dental implant- bone graft at the same time and bone graft with a time of 4 months of integration for the placement of dental implants. For the evaluation of the regenerative effect in the different surgical procedure we interpreted clinical and radiographic controls with the behavior of the marginal bone crest. Histological analyzes of biopsies of regenerated bone were also performed. Results. In the morphological characterization it was found that the materials for regeneration are acellular. The matrix formed by the collagen fibers of the substitute Biomec CX presents an organization and interstitial spaces that allow a good cellular migration. The Biomod product showed a density and particle size suitable for the regenerative process. In the clinical evaluation, all the differences (mesial and distal) of the months evaluated in relation to the bone gain, are statistically significant; T-test, $\mathrm{p}<0.01$ and ANOVA. The average increase in 4 months was $0.235 \mathrm{~mm}$ per mesial $(57.3 \%)$ and $0.237 \mathrm{~mm}$ per distal $(56.7 \%)$. Conclusions. Based on the results obtained both in the morphological characteristics and in the clinical evaluation of the substitutes Biomec CX and Biomod, it was verified that they are suitable materials for use in regenerative processes in which bimaxillary bone lesions are present.
\end{abstract}

Keywords: Bone regeneration, Bone substitution, Osteoconductive, Osteoinductive, Bone injury.

\section{Introducción}

El hueso es un tejido especializado compuesto por células (osteocitos) y componentes extracelulares calcificados, con capacidad para regenerarse tras un trauma o por una injuria controlada. En cualquiera de estos dos casos es común el uso de injertos óseos para la regeneración y formación de hueso nuevo, que llega a ser similar al prexistente (1). Dentro de las alternativas terapéuticas en cirugía oral y maxilofacial en pacientes afectados por lesiones incapacitantes como lo son los procesos infecciosos, osteonecrosis, fracturas patológicas, malformaciones congénitas, secuelas oncológicas, trauma facial, dentoalveolar y reabsorción ósea, se encuentra la utilización de constructos artificiales capaces de inducir la formación de tejido óseo con las mismas características macro y micro morfológicas de cada individuo $(2,3,4)$.

Teniendo como referente clínico que cada defecto óseo tiene un abordaje diferente para cada paciente, es pertinente conocer los materiales de relleno desde el punto de análisis morfológico (forma y tamańo de las partículas), topográfico (características de superficie) y composición porcentual (análisis de compuestos), elementos primordiales para ser considerados como injertos óseos con fines terapéuticos $(5,6)$.

Los injertos óseos cumplen una doble función mecánica y biológica. Inicialmente se debe determinar 
cuál es la función del injerto a utilizar para ejecutar una correcta cirugía ósea regenerativa que pretende regenerar el hueso alveolar perdido por diferentes tipos de secuelas traumáticas. Las interacciones que se presentan entre el injerto óseo y el huésped son de alta complejidad empezando con la adecuada incorporación del injerto y la vascularización de la zona de implantación e inmunogenética entre donante y huésped, pasando por las técnicas y conservación, uso de medicamentos, los factores locales y sistémicos y las enfermedades crónicas degenerativas y terminando en las propiedades mecánicas que dependen de la morfología, topografía y composición del tipo de injerto utilizado, bajo estos principios se han desarrollado diferentes técnicas encaminadas a rellenar los defectos óseos con materiales que induzcan: osteogénesis, osteoinducción y osteoconductividad $(7,8,9)$.

Los injertos óseos se clasifican según la naturaleza del material y a la afinidad entre el ser donante y el ser receptor, de la siguiente manera: autoinjerto (del mismo individuo), aloinjerto (diferente individuo pero misma especie) y xenoinjerto (de especies diferentes). Idealmente un injerto óseo debe tener estas propiedades, además de ser biocompatible y proporcionar estabilidad biomecánica.

El proceso de incorporación de un injerto óseo es un mecanismo complejo que varía dependiendo del sitio de colocación y el tipo de injerto utilizado; está delimitado por:

1. Fase temprana (1 a 3 semanas): osificación membranosa en la zona adyacente a la cortical ósea y conversión del hematoma postoperatorio en estroma fibroblástico alrededor del injerto.

2. Fase intermedia (4 a 5 semanas): incorporación y remodelación del injerto con una zona central cartilaginosa y osificación endocondral alrededor de la misma.

3. Fase tardía (6 a 10 semanas): mayor cantidad de médula ósea en la formación de hueso cortical alrededor de la zona central y remodelación ósea (9).

\section{Materiales y Métodos}

\section{Caracterización morfológica productos Biom $\ c$ Cx y Biomod}

Para la caracterización morfológica se tomaron sustitutos óseos de origen porcino de la casa 3Biomat, para un total de 12 muestras distribuidas en 6 de matriz ósea desmineralizada y liofilizada Biomod, con un tamaño de partícula de 300$600 \mu \mathrm{m}$, Referencia BMD03061, y 6 Membranas de colágeno multilaminar Biomec $\mathrm{Cx}$ con una dimensión de $15 \times 22 \mathrm{~mm}$ barrera media referencia BCX015022BM.

Se realizó un primer análisis de la muestra por medio de estereomicroscopía (HM-LUX 3) con diferentes tipos de aumentos $(4 \mathrm{x}, 10 \mathrm{x}, 40 \mathrm{x})$ y filtros (azul, verde y rojo para longitud de $450 \mathrm{~nm}$, $550 \mathrm{~nm}, 700 \mathrm{~nm}$ ). El análisis morfológico se realizó bajo histología básica, las muestra se fijaron en formol al 10\%. Posteriormente, las muestras se embebieron en parafina, se seccionaron a un espesor de $5 \mathrm{~mm}$ para su posterior coloración con Hematoxilina-Eosina y tricrómico de Masson, se evaluaron bajo microscopia óptica con diferentes aumentos (4x, 40x, 100x, 400x) Nikon 80-I.

El campo óptico de análisis de cada sección incluyo partículas completas de hueso y segmentos iniciales y finales de membranas de colágeno en un área de $1 \mathrm{~mm}$ para 25x, 40x, 1000x y 5000x. Las imágenes fueron digitalizadas y trazadas utilizando el sistema Motic Imagen Plis 2.0 software. Para crear regiones de interés individual.

El análisis estructural se determinó por medio de microscopía de barrido electrónico con aumentos de (400x, 1000x y 5000x) JOEL, modelo JSM 6490-LV, para lo cual las muestras fueron sometidas a técnicas de alto vacío y posterior metalización con partículas de oro, las imágenes capturadas se analizaron por el sistema Motic Imagen Plis 2.0. 
Los análisis químicos de las muestras (composición porcentual) se llevaron a cabo por medio de microanálisis cualitativo y cuantitativo EDS (Detector de Espectroscopía de Disipación de Energía)

La microscopía de barrido electrónico SEM es una técnica de obtención de imágenes con alta resolución y capacidad de analizar características morfológicas, estructurales y químicas de diferentes tipos de muestras.

\section{Evaluación Clínica}

Se realizaron documentos de consentimiento informado para exodoncia dental, sustitutos óseos de origen porcino de la casa 3Biomat, implantes dentales, rehabilitación oral y manejo de material biológico (biopsias).

La actividad clínica se llevó a cabo por medio de un estudio de tipo comparativo y prospectivo con una muestra intencional de 31 pacientes con un total de 83 implantes, con previo requisito de injerto óseo de fémur porcino. Se tuvo en cuenta para las Exodoncia previa dosis antibiótica de 2 gramos de amoxicilina un día antes y durante 4 días más para conseguir dosis plasmáticas adecuadas en el coágulo primario. Previa asepsia y antisepsia del campo operatorio, se realizaron procedimientos quirúrgicos tales como implante pos-exodoncia más injerto óseo, implante dental y en el mismo tiempo quirúrgico, injerto óseo; y por último, injerto óseo con un tiempo de 4 meses de integración para la colocación de implantes dentales. Se hidrata el sustituto óseo con solución salina fisiológica, compactando el material en medianos incrementos dejando fluir el coágulo medular. No se sobre-corrige el defecto para permitir confrontar los tejidos a los cuales se posicionan membranas de colágeno de capa media, con el objetivo de confrontar los tejidos con menos fuerza tensil de las suturas y obtener un mejor selle primario.

Se tomaron biopsias de tejido óseo en la región operatoria por medio de una fresa trefina de 2,3 mm. La muestra biológica se preservó en formol al $10 \%$ para su posterior fijación en parafina; los cortes histológicos fueron teñidos con tinción de hematoxilina-eosina, tinción tricrómica de Masson. Las muestras para microscopía de barrido electrónico fueron metalizadas en oro en alto vacío y analizadas en 400x, 1000x, 5000x para su posterior análisis de EDS (mapping). El detector de espectroscopía por dispersión de energía (EDS) permite además la realización de microanálisis cualitativos y cuantitativos.

La interpretación de los datos fue realizada por medio del programa de digitalización de imágenes Ba210 Motic images Plis 2,0 software. Área de análisis $1 \mathrm{~mm}$ para $25 \mathrm{x}$, lecturas realizadas $25 \mathrm{x}, 40 \mathrm{x}, 100 \mathrm{x}$, 1000x, 5000x.

Se realizaron controles clínicos y radiográficos iniciales a los 2 y 4 meses. Se evaluó al primer y segundo mes la integridad del injerto. Posteriormente, en los controles clínicos se tiene en cuenta, como criterio mayor, el no presentar dehiscencias ni signos de infección pos-quirúrgica.

El método de evaluación se realizó con radiografías peri-apicales con películas de fósforo marca Soderex Imagin Plates, con técnica de paralelismo por medio de aditamentos de la casa Dentsplay. La dosis estimada por placa fue de $7,4 \mu \mathrm{Sv}$ donde se evaluó el comportamiento posicional de la cresta ósea marginal mesial y distal teniendo como referencia la plataforma del implante. Las mediciones fueron tomadas con un calibrador Vernier digital serie 799 con una capacidad de $0-150 \mathrm{~mm}$ y una resolución de $0.01 \mathrm{~mm}$; se realizaron calibraciones cada 10 mediciones. Los datos se confinan en tablas que relacionan longitud del implante, altura de la cresta mesial y distal, tipo de maxilar y datos demográficos de sexo y edad. Se relacionan con criterios de éxito, supervivencia, mal pronóstico y fracaso.

\section{Resultados}

Análisis estadístico. Se utilizó el programa $\mathrm{R}$ versión 3.2.0 de 2015 de la Universidad de Zurich, Suiza. El nivel de significancia se fijó en $p<0,01$. 
Para comparar los cambios en la altura ósea en $\mathrm{mm}$, a diferentes tiempos (Inicial, 2,4 meses) se utilizó la prueba t pareada y para comparar los tres grupos de tratamiento a los dos maxilares se utilizó análisis de varianza (ANOVA).

Los análisis de microscopía muestran características morfológicas y estructurales de partículas córtico-medulares con un tamaño de partícula de $300-600 \mu \mathrm{m}$. Las partículas con morfología cortical aparecen como una masa sólida y continua con cortes regulares bien definidos. Su matriz ósea mineralizada está depositada en laminillas, Figuras 1 y 3.

En las partículas con morfología medular (reticulado, trabeculado), las láminas intersticiales están distribuidas de forma irregular formando placas y trabéculas.

Al examen histomorfométrico todas las áreas de análisis demostraron homogeneidad de las partículas. Las muestras no presentaron ningún tipo de interferencia de materiales. Los análisis se estimaron para $1 \mathrm{~mm}$ en $25 \mathrm{x}, 40 \mathrm{x}, 1000 \mathrm{x}, 5000 \mathrm{x}$.

Las fibras colágenas presentan una estructura secundaria en forma de cadena bien definida. Bajo el análisis de microscopía de barrido electrónico, presenta zonas electro-densas en sus bandas trasversales. Estas regiones contienen más tinción y crean el aspecto bandeado de las fibrillas colágenas.

Todas las muestras son acelulares, no presentan interferencias con otros tipos de materiales, los datos analizados por medio del microanálisis EDS (detector de espectroscopia de disipación de energía) demostró que la composición porcentual de los elementos encontrados en las partículas (calcio, fósforo, oxígeno, carbono) son constituyentes del tejido óseo.

Los datos analizados en las biopsias demostraron que la concentración de calcio es mayor en hueso maxilar superior que en el inferior. Lo mismo es cierto para oxígeno y fosforo pero la concentración de calcio es 7 veces mayor en el maxilar inferior. Para los injertos hay menos calcio y fósforo, pero más oxígeno en el maxilar inferior que en el maxilar superior.

Las fibras colágenas no presentan ningún tipo de partícula interferente, son completamente acelulares y presentan morfología típica de fibras colágenas.

Los datos clínicos demuestran que la cantidad de implantes por paciente es en promedio 2,67, con un rango 1 a 8 implantes, presentando un porcentaje de éxito del $98 \%$ para un total de 83 implantes distribuidos en el maxilar superior (52\%) y 40 implantes en el maxilar inferior (48\%). De los 83 implantes, 1 se catalogó como fracaso.

La Tabla 2 presenta los resultados de altura del hueso crestal. Cada periodo de tiempo está relacionado de mesial a distal, donde al primer mes la cresta mesial presenta un promedio de $0,41 \pm 0,2$ (promedio \pm desviación estándar), a los 2 meses de $0.57 \pm 0,273$ y los 4 meses de $0,645 \pm 0,311$. Los resultados de la cresta distal presentaron al primer mes un promedio de $0,418 \pm 0,22$, a los 2 meses de $0,577 \pm 0,27$ y a los 4 meses de $0,655 \pm 0,311$.

La Tabla 3 presenta los resultados del análisis estadístico expresando la probabilidad de la prueba $t$ pareada, que indica que todas las diferencias fueron significativas para un nivel de significación $p<$ 0,01 . Se muestra también la diferencia entre cada periodo tanto en mm como en porcentaje de cambio respecto al periodo de tiempo anterior.

En la Tabla 4 se relacionan los datos con criterios de éxito, supervivencia, mal pronóstico y fracaso, estimándose como criterios mayores.

Conclusiones estadisticas. Todas las diferencias (mesial y distal) de los meses evaluados son estadísticamente significativas de acuerdo con la prueba $t$ pareada, $p<0,01$. 
El aumento promedio en dos meses fue $0,16 \mathrm{~mm}$ que representa $39 \%$ por mesial y $38 \%$ por distal. El aumento promedio en 4 meses fue $0,235 \mathrm{~mm}$ por mesial $(57,3 \%)$ y $0,237 \mathrm{~mm}$ por distal $(56,7 \%)$ para el total de datos.

El ANOVA para comparar los tres grupos de tratamiento también muestra diferencias significativas.

El grupo con exodoncias tuvo la mayor ganancia para 2 y 4 meses. En la comparación de implantes en maxilar superior no hay diferencias, como tampoco hay diferencias entre mesial y distal, tendiendo a ser mayor la ganancia por distal.

Para comparar los cambios en la altura ósea en $\mathrm{mm}$, a diferentes tiempos (inicial, 2 y 4 meses) se utilizó la prueba t pareada y para comparar los tres grupos de tratamiento a los dos maxilares se utilizó análisis de varianza (ANOVA).

\section{Resultados}

Tabla 1. Descripción demográfica de la muestra.

\begin{tabular}{l|r|r|r|}
\hline & \multicolumn{1}{|l|}{ Hombres } & \multicolumn{1}{l|}{ Mujeres } & \multicolumn{1}{l|}{ Total } \\
\hline $\begin{array}{l}\text { Distribuición } \\
\text { por género }\end{array}$ & 8 & 23 & 31 \\
\hline & $26 \%$ & $74 \%$ & $100 \%$ \\
\hline $\begin{array}{l}\text { Distribuición } \\
\text { por edades }\end{array}$ & Promedio \pm D.S $=42,5 \pm 11,3$ años \\
\hline
\end{tabular}

Tabla 2. Distribución de los implantes.

\begin{tabular}{|c|c|c|c|c|}
\hline \multicolumn{5}{|c|}{ Implantes por paciente $83 / 31=2,67$ Rango: $1-8$} \\
\hline \multicolumn{3}{|c|}{ Maxilar sup. } & 4 & $52 \%$ \\
\hline \multirow[t]{2}{*}{ IMPLANTES } & \multicolumn{2}{|c|}{ Maxilar inf. } & 4 & $48 \%$ \\
\hline & \multicolumn{2}{|c|}{ Total } & 8 & $100 \%$ \\
\hline CIRUGIAS & $\begin{array}{l}\text { Exodoncia } \\
\text { Injerto } \\
\text { Implante }\end{array}$ & $\begin{array}{l}\text { ImpI } \\
\text { Injer }\end{array}$ & & $\begin{array}{l}\text { Injerto } \\
\text { Implante }\end{array}$ \\
\hline Paciente & $10(32 \%)$ & & & $9(29 \%)$ \\
\hline Implantes & $23(27,7 \%)$ & & & $29(35 \%)$ \\
\hline
\end{tabular}

Tabla 3. Resultados altura cresta osea $X \pm S(\mathrm{~mm})$

\begin{tabular}{|l|r|r|}
\hline Tiempo & Mesial & Distal \\
\hline Inicial & $0,41 \pm 0,2$ & $0,41 \pm 0,2$ \\
\hline Dos meses & $0,57 \pm 0,273$ & $0,57 \pm 0,273$ \\
\hline Cuatro meses & $0,645 \pm 0,311$ & $0,645 \pm 0,311$ \\
\hline $\begin{array}{l}\text { Diferencia media } \\
\text { en 2 m }\end{array}$ & $0,16(39 \%)+$ & $0,16(38 \%)$ \\
\hline $\begin{array}{l}\text { Diferencia media } \\
\text { en } 4 \mathrm{~m}\end{array}$ & $0,235(57,3 \%)$ & $0,237(56,7 \%)$ \\
\hline
\end{tabular}

Tabla 4. Comparación de resultados por tipo de tratamiento.

\begin{tabular}{|l|r|r|r|} 
& \multicolumn{1}{|l|}{$\begin{array}{l}\text { Exodoncia } \\
\text { Injerto } \\
\text { Implante }\end{array}$} & $\begin{array}{l}\text { Implante } \\
\text { Injerto }\end{array}$ & \multicolumn{1}{l|}{$\begin{array}{l}\text { Injerto } \\
\text { Implante }\end{array}$} \\
\hline & Mesial & Mesial & Mesial \\
\hline $\begin{array}{l}\text { Diferencia } \\
\text { media en 2 m }\end{array}$ & 0,165 & 0,074 & 0,238 \\
\hline $\begin{array}{l}\text { Diferencia } \\
\text { media en 4 m }\end{array}$ & 0,239 & 0,107 & 0,351 \\
\hline $\begin{array}{l}\text { Diferencia } \\
\text { media en 2 m }\end{array}$ & 0,165 & 0,078 & 0,245 \\
\hline $\begin{array}{l}\text { Diferencia } \\
\text { media en 4 m }\end{array}$ & 0,252 & 0,107 & 0,365 \\
\hline
\end{tabular}
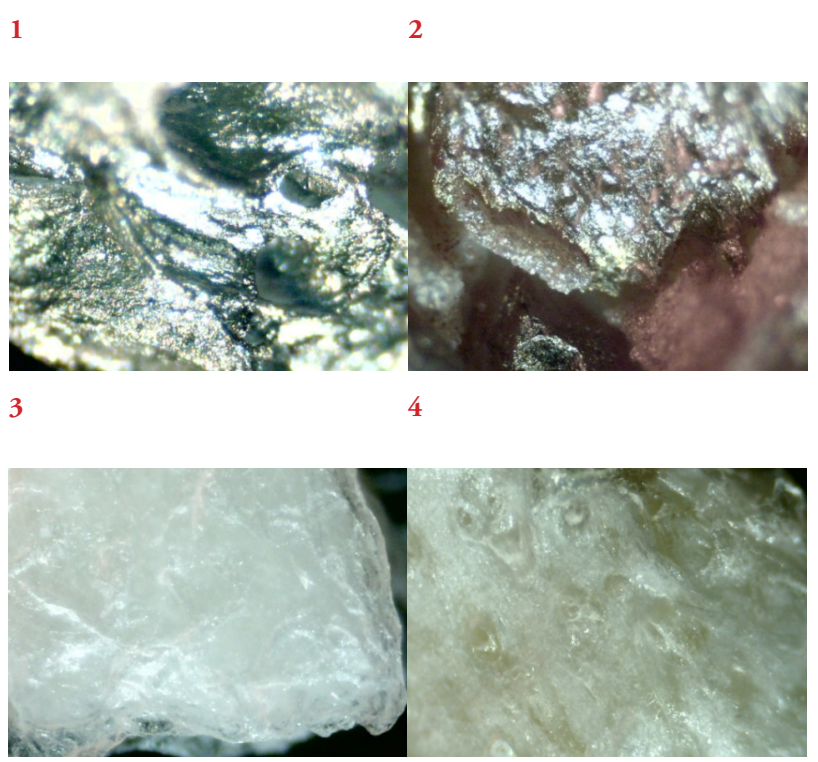

Figura 1. Partículas óseas con técnica de metalización, estereomicroscopía aumento 40x (foto 1 y 2). En las foto 3 y 4 se aprecian las diferencias entre superficie esponjosa y cortical respectivamente 
1

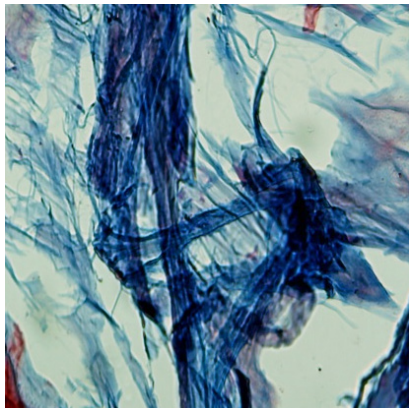

4

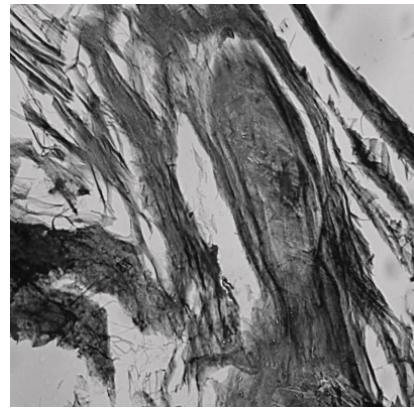

2

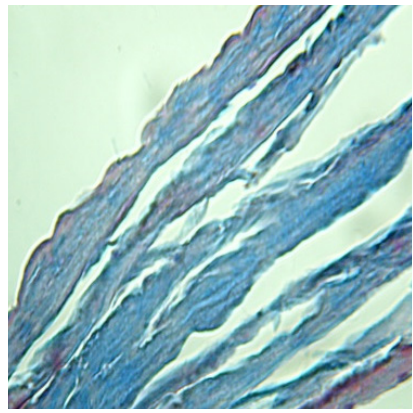

5

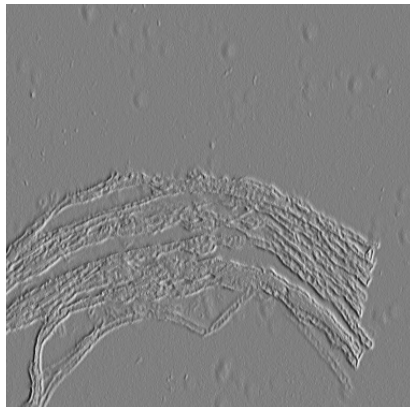

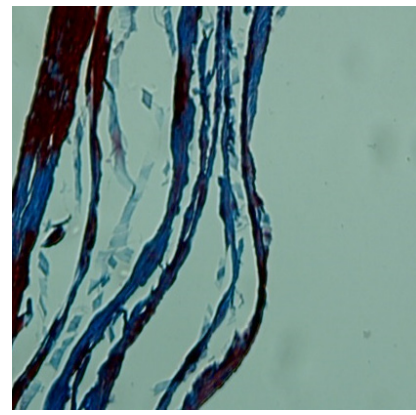

6

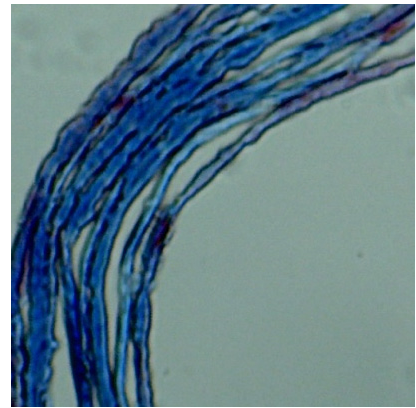

Figura 2. Fibras colágenas, microscopía óptica 40x-100x. Tinción tricrómica de Mansson. Fibras de colágeno que forman enlaces cruzados. Las moléculas de tropocolágeno se polimerizan en sentido longitudinal y lateral, para formar fibrillas reforzadas por numerosos puentes intermoleculares.

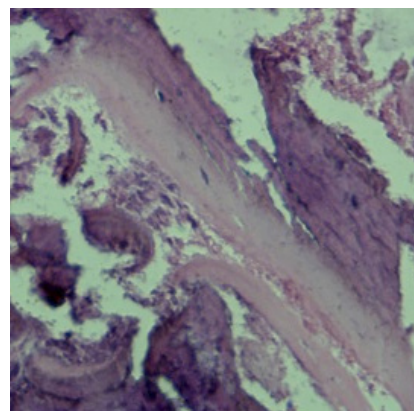

4

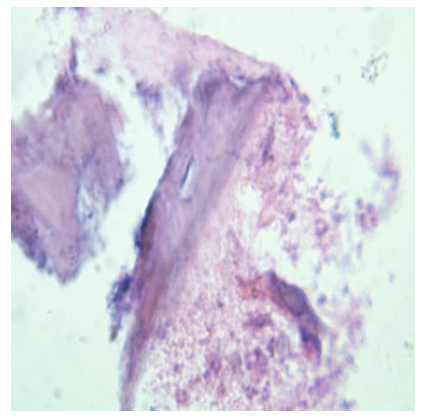

2

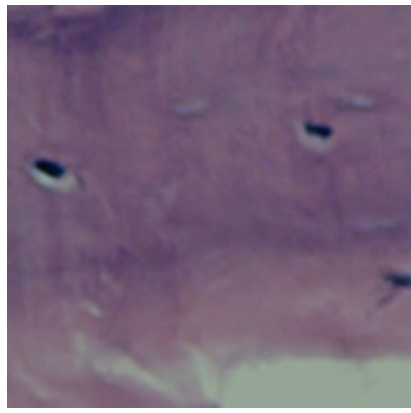

5

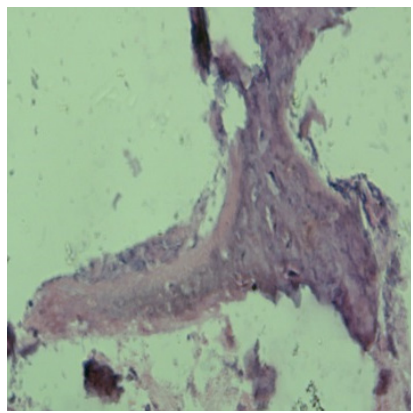

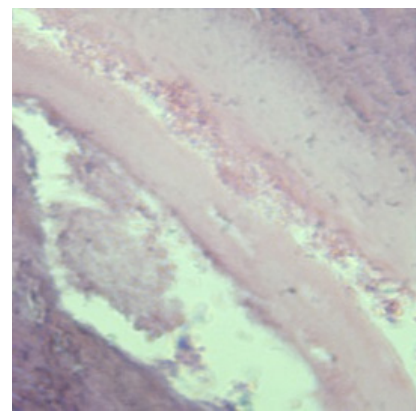

6

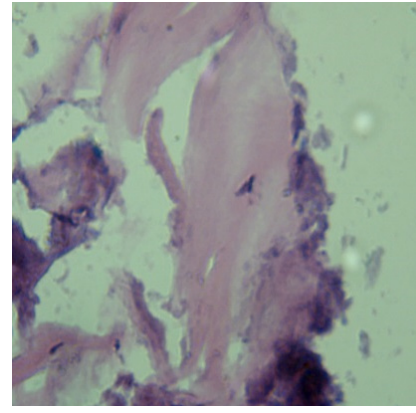

Figura 3. Osteoblastos activos y osteoide H-E corte sin descalcificar tejido humano. Aumento 40x -100x. Estas microfotografías muestran osteoblastos que están depositando en forma activa osteoide sobre una superficie ósea. En las fotos 1 y 6 se observa hueso mineralizado intensamente teñido y osteoide en menor intensidad, producido por la hilera de osteoblastos cuboides. 


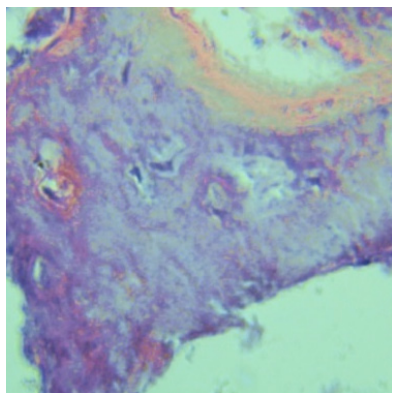

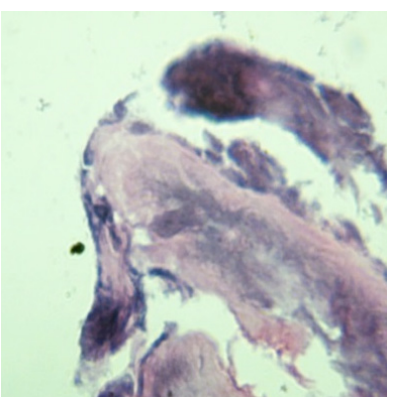

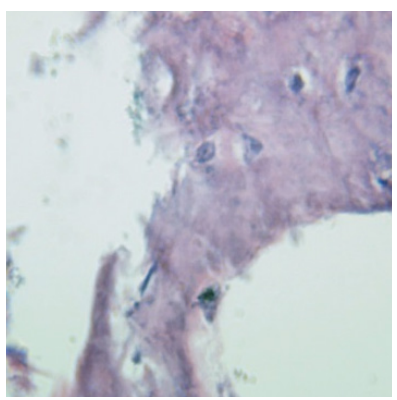

4

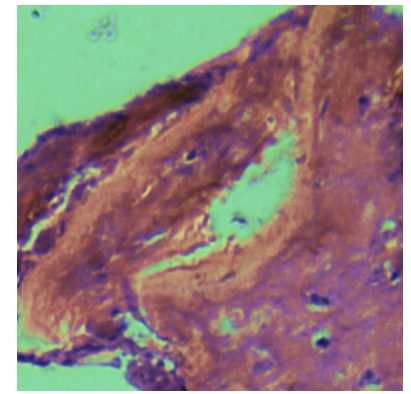

Figura 4. Osteoblastos activos y osteoide H-E corte tejido humano, aumento 40x, 100x. Los osteoblastos activos son células grandes, anchas, de forma fusiforme o cuboides, con abundante citoplasma basófilo que contiene gran cantidad de retículo endoplásmico rugoso. Estas características reflejan la alta tasa de síntesis de proteínas (colágeno Tipo 1).

Tabla 5. Comparación de la composición quimica tisular.

\begin{tabular}{|c|c|c|c|c|}
\hline Descripción & Magnificación & Tamańo (p) & Elemento & Comc (ppm) \\
\hline \multirow{4}{*}{$\begin{array}{l}\text { Hueso humano maxilar inferior } \\
\text { trabeculado medular }\end{array}$} & \multirow{4}{*}{$5000 X$} & \multirow{4}{*}{10} & $\mathrm{CK}$ & 6,30 \\
\hline & & & OK & 6,70 \\
\hline & & & PK & 4,90 \\
\hline & & & $\mathrm{CaK}$ & 1,00 \\
\hline \multirow{4}{*}{ Hueso humano maxilar superior } & \multirow{4}{*}{$5000 X$} & \multirow{4}{*}{10} & CK & 8,70 \\
\hline & & & OK & 9,00 \\
\hline & & & PK & 4,10 \\
\hline & & & $\mathrm{CaK}$ & 7,00 \\
\hline \multirow{4}{*}{ Injerto maxilar inferior } & \multirow{4}{*}{$5000 X$} & \multirow{4}{*}{10} & CK & 8,43 \\
\hline & & & OK & 4,59 \\
\hline & & & $\mathrm{PK}$ & 10,53 \\
\hline & & & $\mathrm{CaK}$ & 12,36 \\
\hline \multirow{4}{*}{ Injerto maxilar superior } & \multirow{4}{*}{$5000 X$} & \multirow{4}{*}{10} & $\mathrm{CK}$ & 8,22 \\
\hline & & & OK & 7,60 \\
\hline & & & PK & 8,40 \\
\hline & & & $\mathrm{CaK}$ & 12,22 \\
\hline \multirow{4}{*}{ Particula } & \multirow{4}{*}{$5000 X$} & \multirow{4}{*}{10} & $\mathrm{CK}$ & 7,83 \\
\hline & & & OK & 6,14 \\
\hline & & & PK & 11,60 \\
\hline & & & $\mathrm{CaK}$ & 12,60 \\
\hline \multirow{2}{*}{ Fibra colágena nativa } & \multirow{2}{*}{$5000 X$} & \multirow{2}{*}{10} & $\mathrm{CK}$ & 67,22 \\
\hline & & & OK & 0,47 \\
\hline \multirow{2}{*}{ Producto dos (2) meses } & \multirow{2}{*}{$5000 X$} & \multirow{2}{*}{10} & $\mathrm{CK}$ & 98,31 \\
\hline & & & OK & 13,18 \\
\hline \multirow{2}{*}{ Laboratorio cuatro (4) meses } & \multirow{2}{*}{$5000 X$} & \multirow{2}{*}{10} & $\mathrm{CK}$ & 89,16 \\
\hline & & & $\mathrm{OK}$ & 2,13 \\
\hline
\end{tabular}

Comparación de la composición química tisular entre humano de maxilar superior e inferior Vs. sustitutos óseos Biomec CXy Biomod. Se comprobó que son materiales aptos para su uso en procesos regenerativos en los que se presenten lesiones óseas bimaxilares. 

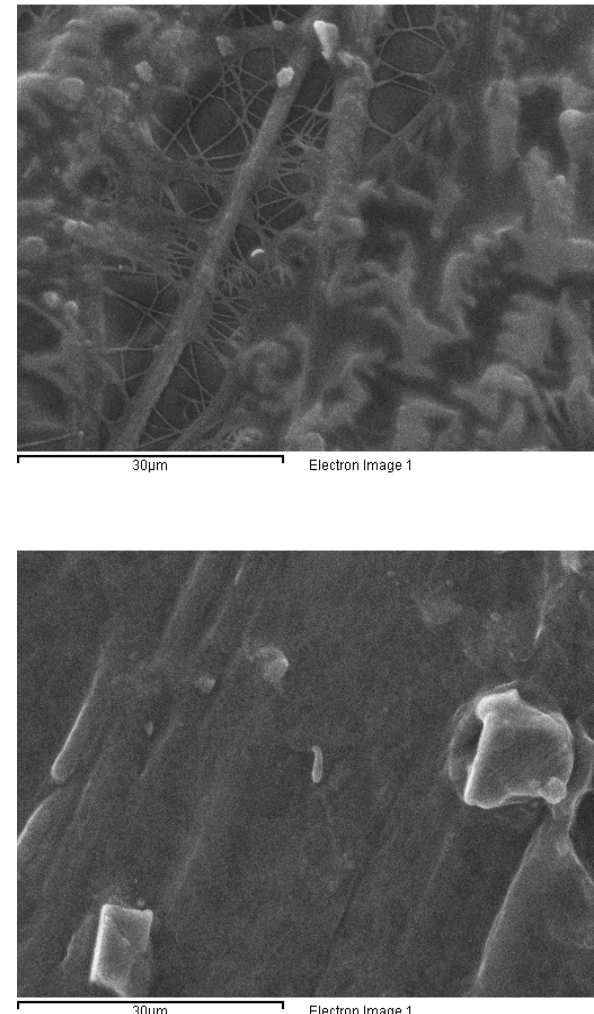
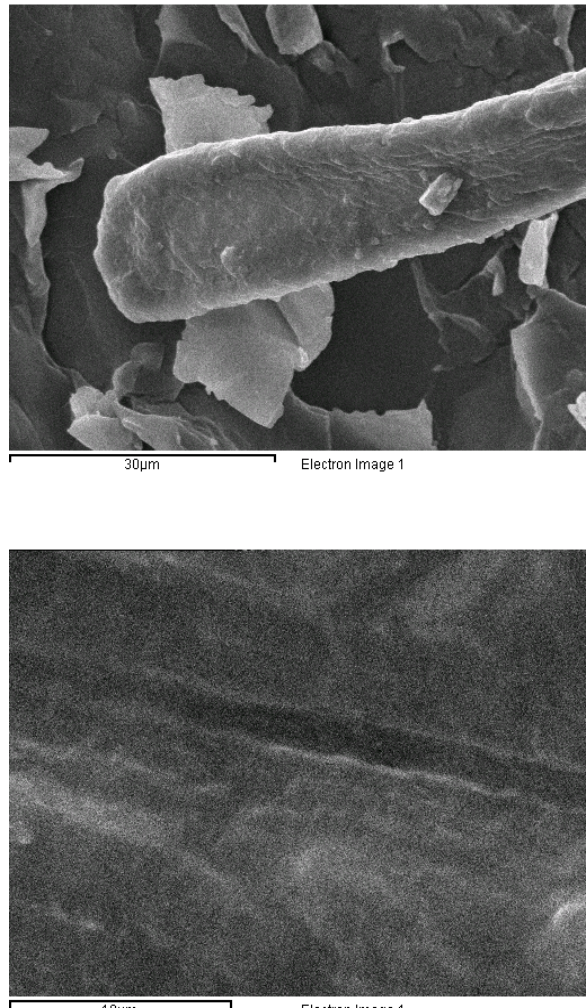

$10 \mu \mathrm{m}$

Electron Image 1

Figura 5. Micrografías electrónicas. Aumento 3000x, coloración tricrómica de Mansson. Las moléculas de colágeno se agregan para formar las fibrillas con sus bandas transversales. Las lagunas o agujeros contienen más tinción y crean el aspecto bandeado de las fibrillas colágeno. Se puede observar variabilidad en los patrones de orientación, tamańo y densidad. Las fibras de colágeno son grandes, se disponen densamente agrupadas y orientándose en una dirección para conseguir la máxima resistencia a la tracción.

1

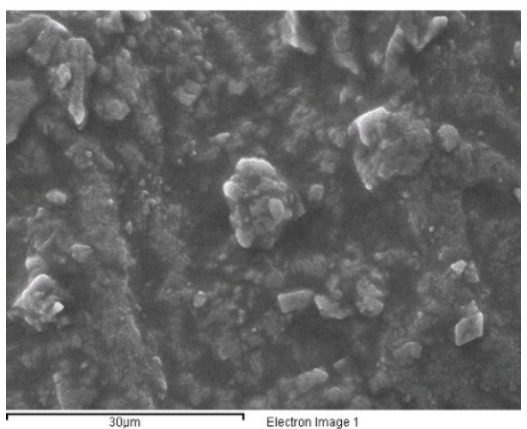

2

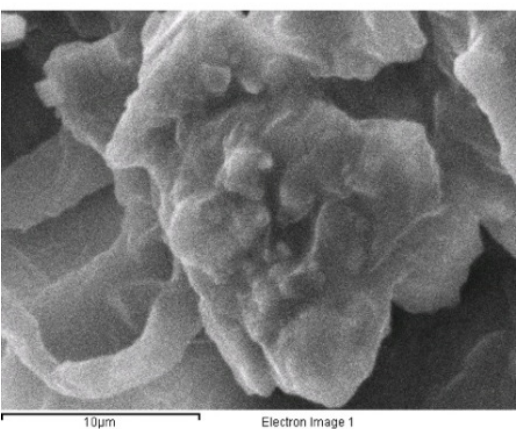

3

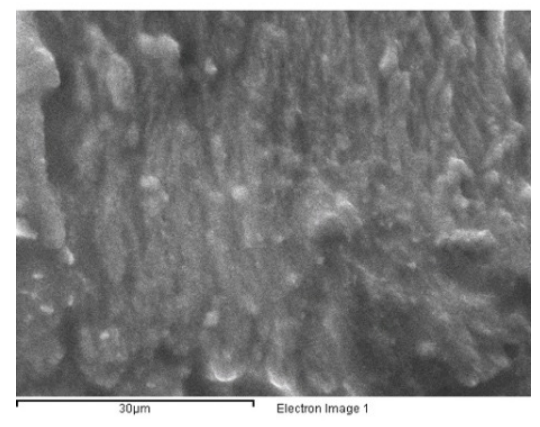

Figura 6. Micrografías de barrido electrónico aumento 3000x para las fotos 1 y 3 y 5000x para la foto 2.

Se observan características morfológicas de partículas de origen medular (foto 1) y cortical (foto 3 ). 

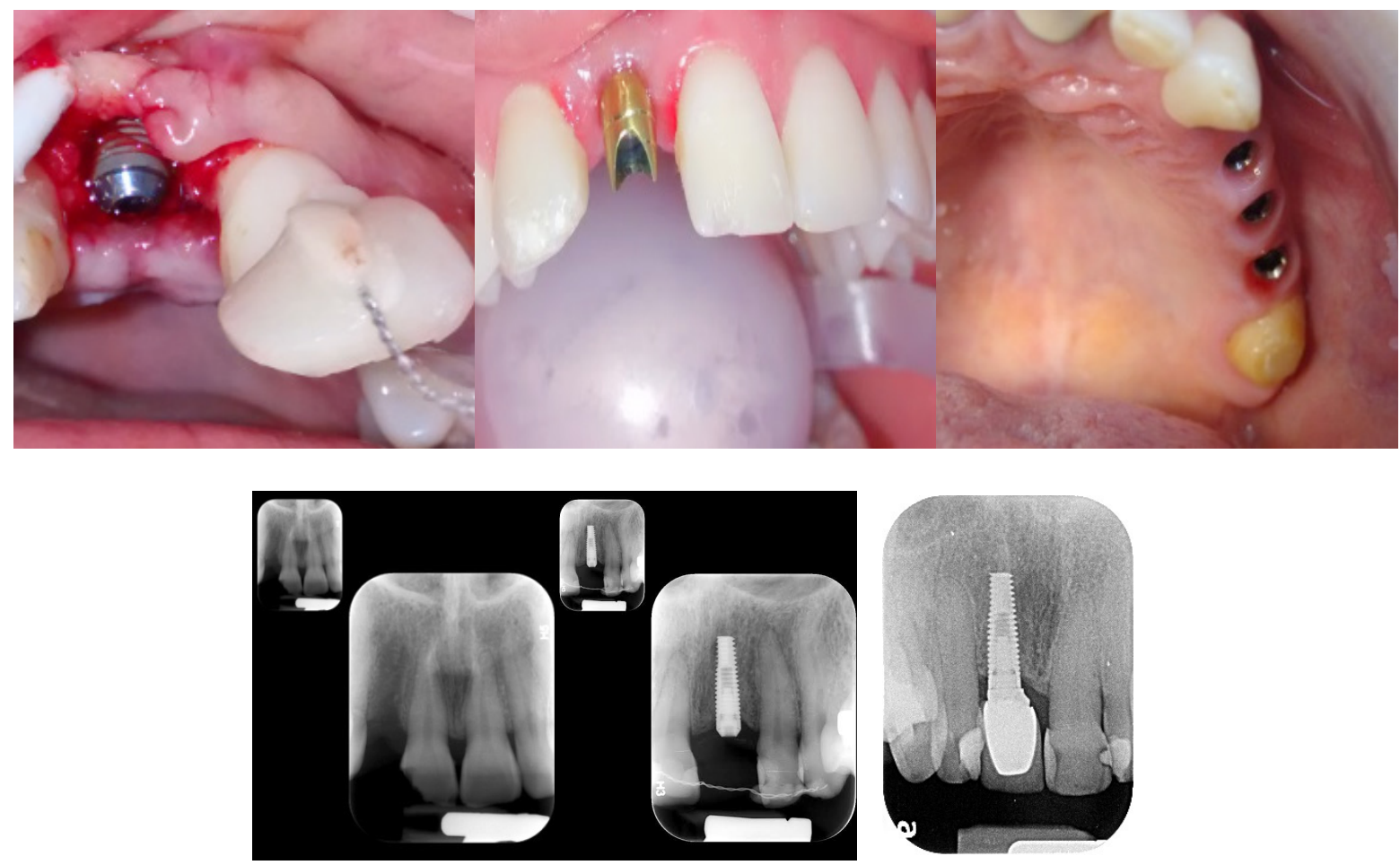

Figura 7. Controles clínicos y radiográficos (inicial 2 meses y 4 meses respectivamente para evaluar el comportamiento de la cresta ósea maginal). Se muestran tiempos adecuados de oseointegración.

\section{Discusión}

La regeneración de defectos óseos causados por traumas faciales, dento-alveolares, enfermedades periodontales, iatrogenias, secuelas oncológicas, malformaciones congénitas o simplemente la pérdida de la altura ósea por mecanismos de reabsorción, durante décadas ha sido un reto para los investigadores que buscan la rehabilitación estética y funcional de estos defectos en el paciente.

Los sustitutos óseos estudiados demostraron cumplir con características que son importantes a la hora de escoger un material para procesos regenerativos. Entre estas encontramos que al ser dispositivos de origen xenogénico, estos deben presentar una baja o nula celularidad, lo que permite que no se generen reacciones inmunogénicas no deseadas en el organismo receptor. Por otro lado, el producto Biomec CX que se caracteriza por ser un dispositivo compuesto en su mayoría por colágeno, presentó una estructura bien definida, de aspecto bandeado típico de fibras colágenas. Esto facilita que se dé una adecuada migración de células como los fibroblastos que son importantes en los procesos de regeneración ya que se encargan de la liberación de factores de crecimientos importantes en los procesos de osteoinducción y osteogénesis. El producto Biomod que está compuesto por partículas de hueso córtico-esponjoso de fémur porcino mostró una adecuada distribución de las partículas que van de 300 a 600 micras, y una morfología continua. Esto es importante ya que es necesario que los procesos de reabsorción de los injertos sean eficientes, para que los tejidos en neoformación correspondan a lo deseado.

Una vez se conocen las propiedades de los materiales que se desean utilizar en procesos de regeneración es importante que el especialista en su práctica clínica realice un adecuado control y monitoreo del paciente teniendo en cuenta un apropiado diagnóstico inicial, antecedentes sistémicos del pacien- 
te, aplicación adecuada de protocolos quirúrgicos, compatibilidad de materiales, microambiente celular, tiempos adecuados de integración biológica, condiciones de asepsia y antisepsia, y seguimiento pos-operatorio; ya que todas esta variables pueden representar el éxito o fracaso de una adecuada regeneración tisular $(10$ - 16).

Luego de realizar el procedimiento de injertar un material de regeneración, varios autores han propuesto un límite de $1,5 \mathrm{~mm}$ de pérdida ósea alrededor de implantes osteointegrados exitosamente durante el primer ańo de carga y un límite para los siguientes ańos de $0,2 \mathrm{~mm}(10,11,2,17,18$, 19). Recientemente el consenso del Congreso Internacional de Implantólogos orales (20), aprobó cuatro categorías clínicas que definen las condiciones de éxito, supervivencia y fracaso de los implantes dentales. Allí se estableció que un implante se clasifica en grupo de éxito cuando cumple las siguientes condiciones: ausencia de dolor a la palpación, percusión o función, sin movilidad clínica en cualquier dirección, ausencia de exudado y pérdida ósea marginal radiográfica menor de $2 \mathrm{~mm}$.

Los implantes evaluados en este estudio y su directa relación con la cresta ósea se puede catalogar en el grupo de éxito a los 4 meses. Estos datos determinan el éxito de la futura carga funcional mostrando una marcada estabilización de la anatomía peri-implantar. Arismendi JA en su estudio comparativo de implantes de superficie lisa y rugosa, resultado a 36 meses, determinó que los implantes de superficie lisa con carga funcional presentaron una pérdida ósea de $0,4 \mathrm{~mm}$, mientras que a los 24 y 36 meses la pérdida ósea fue de $1,5 \mathrm{~mm}$. Igualmente los implantes de superficie rugosa con carga funcional a los 12 meses, demostraron pérdida ósea promedio de $0,6 \mathrm{~mm}$. A los 24 meses dicha pérdida incrementó en promedio $1,4 \mathrm{~mm}$, y a los 36 meses se aumentó ligeramente a $1,5 \mathrm{~mm}$ en promedio.

Chang y Wennstrom (21), estudiaron 18 pacientes con un total de 43 implantes sin ningún tipo de in- jerto, evaluando las alteraciones del hueso crestal y su relación con tejidos blandos durante 3 años. En los 6 meses después de la cirugía de colocación de implantes de una etapa, el margen del tejido blando retrocedió aproximadamente $0,6 \mathrm{~mm}$ y $1,1 \mathrm{~mm}$ sin reportes significativos. Durante los meses siguientes estos mismos autores reportan un estudio evaluado en 5 ańos con controles radiográficos analizando la topografía del hueso proximal en unidades de diente/implante con un $n=36$ comparados con implante/implante con un $n=67$ y un $p<0,052$. Los resultados reportados muestran que en las unidades de diente/implante la pérdida de hueso proximal era de $0,4 \mathrm{~mm}$ y en las unidades de implante/implante es de $0,5 \mathrm{~mm}$, demostrando una mayor pérdida ósea en el espacio de interimplantación. En el presente estudio se evaluaron 83 implantes, se realizaron procedimientos quirúrgicos tales como implante pos-exodoncia más injerto óseo, implante dental y en el mismo tiempo quirúrgico, injerto óseo, y por último, injerto óseo con un tiempo de 4 meses de integración para la colocación de implantes dentales Todas las diferencias (mesial y distal) de los meses evaluados son estadísticamente significativas según la prueba $t$ pareada, $p<0,001$ y ANOVA. El aumento promedio en 4 meses fue $0,235 \mathrm{~mm}$ por mesial $(57,3 \%)$ y $0,237 \mathrm{~mm}$ por distal $(56,7 \%)$.

Lars Schropp (15) realizó un estudio comparando los cambios del hueso crestal después de implantes pos-exodoncia contra implantes de 3 meses después de la exodoncia, en 46 pacientes relacionados en dos grupos, $n=23$, realizando controles clínicos y radiográficos. Los resultados demuestran una supervivencia del $91 \%$ en la implantación inmediata con una pérdida ósea de $2 \mathrm{~mm}$, un $96 \%$ en el grupo de 3 meses y una pérdida ósea de 1,5 mm en promedio. En el presente estudio se presentó éxito del $90 \%$ con una altura promedio de la cresta ósea de $1 \mathrm{~mm}$ con varianzas homogéneas y pequeñas entre $0,18 \mathrm{~mm}$ y $0,22 \mathrm{~mm}$ demostrado como injertos previos no solamente son rellenos de cavidades, sino elementos osteoconductivos que asociados a implantes colocados infracrestalmente promueven una nueva formación de hueso crestal. 
Los resultados sobre pérdida ósea ocurrida en estudios $(2,6)$ que evalúan el comportamiento de implantes del sistema Branemark después del primer ańo de carga funcional, muestran que la disminución ósea se encuentra entre 0,4 y 1,6mm con promedio de $0,93 \mathrm{~mm}$. Los resultados obtenidos en esta investigación se encuentran en un promedio de $0,18 \mathrm{~mm}$ con un aumento de la altura ósea en segundo mes de 0,16 mm que representa 39\% por mesial y $38 \%$ por distal. El aumento promedio en 4 meses fue $0,235 \mathrm{~mm}$ por mesial $(57,3 \%)$ y $0,237 \mathrm{~mm}$ por distal $(56,7 \%)$ para el total de datos.

La composición de hueso y membrana de origen porcino de la casa 3Biomat es morfológicamente similar a la del hueso humano en el maxilar superior/ maxilar inferior. Los resultados clínicos demostraron tener una ganancia alrededor de la cresta ósea. Es importante resaltar que este material no presentó reacción a cuerpo extraño, poca reacción inflamatoria, no se presentó dehiscencia de la herida quirúrgica en ninguno de los casos clínicos; elementos importantes de biocompatibilidad (22 - 24).

La capacidad osteoconductiva, se demostró desde el inicio de la caracterización de los materiales estimada por su morfología y estructura regular, tamaño de partícula, su superficie de contacto córtico-medular y la interconexión entre poros los cuales determinan una respuesta biológica de crecimiento y aposición celular, elementos importantes en la formación de tejido óseo neoformado $(25-28)$.

\section{Conclusiones}

- A partir de los resultados obtenidos tanto en las características morfológicas como en la evaluación clínica de los sustitutos óseos Biomec Cx y Biomod, se comprobó que son materiales aptos para su uso en procesos regenerativos en los que se presenten lesiones óseas bimaxilares.

- Los sustitutos óseos Biomec Cx y Biomod son materiales biocompatibles con mínima respuesta inflamatoria. En ninguno de los tiempos evaluados se presentó reacción a cuerpo extraño.
- Los sustitutos óseos Biomec Cx y Biomod favorecen el proceso normal de regeneración ósea comportándose como un material osteoconductivo que permite el crecimiento óseo en sentido horizontal y vertical.

- El tiempo de reabsorción está ubicado en 4 meses, periodo analizado en este estudio.

\section{Referencias}

1. Wheater, Barbara Young, Geraldine O’Dowd. Histología Funcional: sexta edición Elsevier 2014;180-192.

2. Arismendi JA, Mesa AL, García LP, Salgado JF, Castaño C, Mejía R. Estudio comparativo de implantes de superficie lisa y rugosa. Resultados a 36 meses. Rev. Fac Odontol Univ Antioq 2010;21(2):159-169.

3. EspositoM, GrusovinMG, Worthington HV. Interventions for replacing missing teeth: treatment of peri-implantitis. Cochrane Database Syst. Rev. 2012 Jan 18.pub 5.

4. Soardi CM, Bianchi AE, Zandanel E, Spinato S. Clinical and radiographic evaluation of immediately loaded one-piece implants placed into fresh extraction sockets. Quintessence Int. 2012 Jun;43(6):449-56.

5. Rodríguez E, Pinzón L, Garzón D. Análisis Histológico de implantes de material poroso de apatita carbonatada de síntesis seca y células de medula ósea en un modelo porcino. Revista Med 2010

6. Arismendi JA, Castaño AC,Mejia RM,Mesa AL, Castañeda DA, Tobon SI. Evidencia de cambios clínicos y radiográficos en implantes oseointegrados de superficie maquinada y modificada, 3 y 6 meses de seguimiento. Rev Fac Odontol Univ Antioq 2006;18(1):6-16.

7. Whang PG, Wang JC. Bone graft substitutes for spinal fusion. Spine J 2003;3:155-165.

8. Stevenson S. Biology of bone grafts. Orthop Clin North Am 1999;30:543-552.

9. Boden SD. The biology of posterolateral lumbar spinal fusion. Orthop Clin North Am 1998;29:603-619

10. Gazdag AR, Lane JM, Glaser D, Forster RA. Alternatives to autogenous bone graft: efficacy and indications J Am Acad Orthoped Surg 1995;3:1-8.

11. Maté-Sánchez de Val JE, Mazón P, Guirado JL, Delgado RA, Piedad M, Fernandez R, Negri B, Abboud M, De Aza P. Comparasion of three hydroxyapatite/b-tricalcium phosphate/ collagen ceramic scaffolds: Anin vivostudy, Journal of Biomedical Materials Research Part A, 2014 
12. Caubet J, Ramis J, Ramos-Murguialday M, Morey M, Monjo M. Gene expression and morphometric parameters of human bone biopsies after maxillary sinus floor elevation with autologous bone combined with Bio-Oss ${ }^{\oplus}$ or BoneCeramic ${ }^{\oplus}$, Clinical Oral Implants Research, 2014.

13. Sartori, G. Giavaresi, M. Tschon, L. Martini, L. Dolcini, M. Fiorini, D. Pressato, M. Fini, Long-term in vivo experimental investigations on magnesium doped hydroxyapatite bone substitutes, Journal of Materials Science: Materials in Medicine, 2014

14. Sartori, G. Giavaresi, M. Tschon, L. Martini, L. Dolcini, M. Fiorini, D. Pressato, M. Fini, Long-term in vivo experimental investigations on magnesium doped hydroxyapatite bone substitutes, Journal of Materials Science: Materials in Medicine, 2014

15. Fischer, S. Fickl, Knochenersatzmaterialien zur Sinusbodenelevation, Der Freie Zahnarzt, 2012, 6, 89

16. Lars L Schropp, Lambros L Kostopoulos, and Ann A Wenzel. Bone healing following immediate versus delayed placement of titanium implants into extraction sockets: a prospective clinical study. J Oral Maxillofac Implants 18(2):189-99 (2003).

17. Esposito M, Maghaireh H, Grusovin MG, Ziounas I, Worthington HV. Soft tissue management for dental implants: what are the most effective techniques? A Cochrane systematic review. J Oral Implantol. 2012 Autumn;5(3):221-38.

18. Bressan E. Nanostructured Surfaces of Dental Implants. J. Mol. Sci. 2013, 14, 1918-1931.

19. Chang M, Wennström JL. Soft tissue topography and dimensions lateral to single implant-supported restorations. A cross-sectional study. Clin Oral Implants Res. 2013 May;24(5):556-62.

20. Chang M, Wennström JL. Peri-implant soft tissue and bone crest alterations at fixed dental prostheses: a 3-year prospective study. Clin Oral Implants Res. 2010 May;21(5):52734 .

21. Chang M, Wennström JL. Bone alterations at implant-supported FDPs in relation to inter-unit distances: a 5-year radiographic study. Clin Oral Implants Res. 2010 Jul;21(7):735-40.

22. Chang M, Wennström JL. Longitudinal changes in tooth/ single-implant relationship and bone topography: an 8-year retrospective analysis. Clin Implant Dent Relat Res. 2012 Jun;14(3):388-94.

23. Gierse H, DonathK. Reactions and complications after the implantation of Endobon including morphological examination of explants. Archives of Orthopaedic and Trauma Surgery 1999;119: 349-355.

24. Tamai N, Myoui A, TomitaT, Nakase T, TakanaJ, Ochi T, YoshikawaH. Novel hydroxyapatite ceramics with an interconnective porous structure exhibit superior osteoconduction in vivo. Journal of Biomedical Materials Research 2002; 59: 110-117.
25. Khodadadyan-Klostermann C, Liebi T, Melcher I, Raschke $\mathrm{M}, \mathrm{Haas} \mathrm{N}$. Osseous integration of hydroxyapatite grafts in metaphyseal bone defects of the proximal tibia (CT-study). Acta Chirurgiae Orthopaedicae et Traumatologiae Cechoslovaca 2002;69: 16-21.

26. MotomiyaM, Ito M, TakahataK, Irie K, Abumi K, Minami A. Effect of hydroxylapatite porous characteristics on healing outcomes in rabbit posterolateral spinal fusion model. European Spine Journal 2007;16: 2215-2224.

27. Nausa, J.G. Evaluación clínica y radiográfica de injertos biocerámicos tipo hidroxiapatita como alternativa en la reconstrucción de alveolos dentarios postexodoncia. NOVA 2014; 12 (22): 157 -164 .

28. Flórez, R. A. N. Avances y perspectivas en Síndrome de Asperger. 2014; Nova, 12(21). 